\title{
Análise da Influência da Bandagem Funcional de Tornozelo no Tempo de Reação do Fibular Longo em Sujeitos Saudáveis
}

\author{
Analysis of Ankle Taping Influence in the Peroneal Reaction Time in \\ Healthy Subjects
}

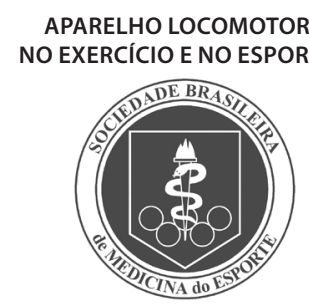

Artigo Original
Maurício Couto Meurer Adriana Moré Pacheco² Ivan Pacheco 3

Marcelo Faria Silva ${ }^{4}$

1. Fisioterapeuta da Clínica SOS Esportes - Porto Alegre, RS; PósGraduado no Curso de Cinesiologia do Programa de Pós-Graduação em Ciências do Movimento Humano/ UFRGS.

2. Fisioterapeuta. Doutora em Ciências do Movimento Humano/ UFRGS; Professora do Curso de Fisioterapia da Universidade Federal do Rio Grande do Sul (UFRGS).

3. Ortopedista e Traumatologista do Esporte da Clínica SOS

Esportes - Porto Alegre, RS; Doutor em Ciências do Movimento Humano/UFRGS, Ortopedista e Traumatologista do Esporte do Clube Grêmio Náutico União Porto Alegre, RS; Diretor Médico da Federação Gaúcha de Futebol.

4. Professor do Curso de Fisioterapia do Centro Universitário Metodista IPA - Porto Alegre, RS; Mestre em Ciências do Movimento Humano/ UFRGS; Doutorando em Ciências do Movimento Humano/UFRGS.

\section{Endereço para correspondência:} Rua Pedro Chaves Barcelos, 585, B. Mon't Serrat - Porto Alegre, RS. E-mail:

mauricio_meurer@yahoo.com.br

\begin{abstract}
RESUMO
Objetivos: O objetivo deste estudo foi analisar a influência da bandagem funcional de tornozelo no tempo de reação do fibular longo de indivíduos sadios. Métodos: A amostra foi composta por oito indivíduos do gênero masculino, fisicamente ativos, sem histórias prévias de lesões de tornozelo e, com os resultados negativos aos testes de instabilidade articular de tornozelo. Foram usados como instrumentos da pesquisa, a bandagem funcional de tornozelo tipo bota fechada, para realizar o teste uma plataforma capaz de inclinar $30^{\circ}$ no plano frontal simulando a entorse lateral do tornozelo e um eletromiógrafo de oito canais (Bortec Electronics Incorporation, Canadá) para aquisições dos sinais eletromiográficos. Os sinais EMG foram coletados juntamente com o sinal da plataforma (sincronismo). A coleta dos dados foi realizada em dois momentos, primeiramente sem a bandagem e logo após com a bandagem. Resultados: Os indivíduos tinham idade média de 23,3 anos ( $\pm 2,8)$, massa corpórea de $74,4 \mathrm{~kg}( \pm 9,4)$, estatura de $1,7 \mathrm{~m}( \pm 0,4)$ e IMC de $23,7 \mathrm{~kg} / \mathrm{m}^{2}( \pm 2,4)$. O estudo encontrou diferença estatisticamente significativa $p=0,018$ na situação da bandagem funcional de tornozelo com diminuição do tempo de reação do fibular longo. Conclusão: Acreditamos que esta melhora se deva ao íntimo contato da bandagem com os receptores cutâneos do tornozelo, aumentando a ativação do reflexo fibular, com consequente ganho proprioceptivo e, assim, aumentando a habilidade dos sujeitos em responder a situações súbitas de entorses. O estudo demonstrou diminuição no tempo de reação do fibular longo de indivíduos sadios com o uso da bandagem funcional de tornozelo quando submetidos à inclinação lateral súbita do tornozelo.
\end{abstract}

Palavras-chave: tornozelo, bandagem, tempo de resposta eletromiográfica.

\begin{abstract}
Objective: The aim of this study was to analyze the influence of the ankle functional tape on long fibular reaction time in health subjects. Methods: The sample was composed of eight physically active male subjects, without ankle injury history and with negative results on ankle joint instabilities tests. The instruments used in this research were an ankle functional tape(closed boot type), a platform capable to $30^{\circ}$ frontal inclination and an eight-channel electromyograph (Bortec Electronics Incorporation, Canada) for the electrical signals acquisition. The EMG signals were collected concomitantly to the platform (synchronism). Data were collected in two moments: firstly without tape and immediately after, with the tape. Results: The subject average age was 23.3 years $( \pm 2.8)$, body mass of $74.4 \mathrm{~kg}( \pm 9.4)$, height of 1.7 $\mathrm{m}( \pm 0.4)$ and BMl of $23.7 \mathrm{~kg} / \mathrm{m}^{2}$. The research found statistically significant difference of $p=0.018$ when the subjects were using the functional ankle tape, with a decreasing of reaction time in long fibular. Conclusion: We believe that this increase is related to the close contact of the functional tape with the ankle cutaneous receptors, increasing the fibular reflex activation, with a consequent proprioceptive gain and hence increasing the subjects' ability to respond to sudden ankle sprains. The study showed decrease in long fibular reaction time of health subjects while using this kind of ankle tape when submitted to a sudden lateral ankle inclination.
\end{abstract}

Keywords: ankle, bandage, electromyographic response time. 


\section{INTRODUÇÃO}

A alta incidência das lesões ligamentares de tornozelo assumem maior importância na prática clínica a cada dia, podendo causar incapacidade imediata ou tardia. Dados epidemiológicos apontam esta lesão como uma das mais frequentes entre os atletas e, na população geral, incide na taxa de 1:10.000 indivíduos/dia ${ }^{(1)}$.

Aproximadamente, 80 a 90\% das entorses de tornozelo ocorrem no compartimento lateral da articulação como resultado do mecanismo de flexão plantar e inversão(2). O mecanismo de entorse lateral de tornozelo é relatado quando há supinação excessiva do retropé combinado com rotação externa da tíbia no início do contato do pé com o solo durante a marcha, corrida ou salto ${ }^{(3)}$. A entorse de tornozelo caracteriza-se como uma lesão na qual vários tecidos são estirados e/ ou rompidos ${ }^{(4,5)}$

O mecanismo de inversão ocorre em grande velocidade, não permitindo, muitas vezes, que o músculo reaja a tempo de estabilizar a articulação, impondo, assim, sobrecarga lesiva ao complexo ligamentar lateral(6), atingindo, principalmente, os músculos fibulares curto e longo(4). Com esse estiramento, pode ocorrer alteração da capacidade proprioceptiva pela lesão e pela instabilidade articular(4,5,7-11).

Em virtude da alta incidência de entorses de tornozelo, foram desenvolvidas estratégias preventivas, sendo a bandagem funcional de tornozelo amplamente utilizada como uma das técnicas para prevenir as recidivas de entorse ${ }^{(12-14)}$. Entretanto, existe carência de pesquisas com o uso da bandagem em indivíduos saudáveis e com o aumento da aplicação da técnica por fisioterapeutas dentro de seus consultórios e clínicas, sentimos a necessidade de explorar este assunto em indivíduos sadios.

Garrick et al. ${ }^{(15)}$ documentaram a eficácia da bandagem de tornozelo em reduzir a incidência de entorse e outras pesquisas avaliaram a capacidade de contenção mecânica excessiva que a fita adesiva ou bandagem tem de fornecer aos movimentos do tornozelo $(12,13,16,17)$. Os efeitos da bandagem de tornozelo na entrada proprioceptiva ao sistema nervoso central(18), a atividade dos fibulares ${ }^{(12,13,16)}$ e a contenção do movimento excessivo do tornozelo ${ }^{(2)}$ aliados à limitação da inversão, são muito importantes na prevenção da entorse. Autores relataram um retardo do tempo de reação da musculatura fibular após entorse de tornozelo ${ }^{(13,19-21)}$

É discutido que, devido ao contato íntimo da bandagem com os receptores cutâneos, possa ocorrer um estímulo e assim tem-se uma maior ativação do reflexo de estiramento fibular, melhorando a propriocepção do tornozelo(23). Sendo assim, o objetivo deste estudo foi analisar a influência da bandagem funcional de tornozelo no tempo de reação do músculo fibular longo de indivíduos sadios.

\section{MÉTODOS}

\section{Amostra}

A amostra foi constituída por oito indivíduos sadios, do gênero masculino, praticantes de atividade física regular (mínimo de duas vezes por semana), sem história prévia de lesões no tornozelo analisado e com resultados negativos para os testes de instabilidade articular do tornozelo (gaveta talar anterior e o teste de hiperlassidão ligamentar de tornozelo). Sendo escolhida a perna dominante para o teste.

\section{Instrumentos de pesquisa e caracterização dos equipamentos Bandagem funcional de tornozelo}

Bandagem do tipo esparadrapo não-elástico que continha em sua composição borracha natural e óxido de zinco, o que proporciona boa aderência e flexibilidade ao tecido (Figura 1).

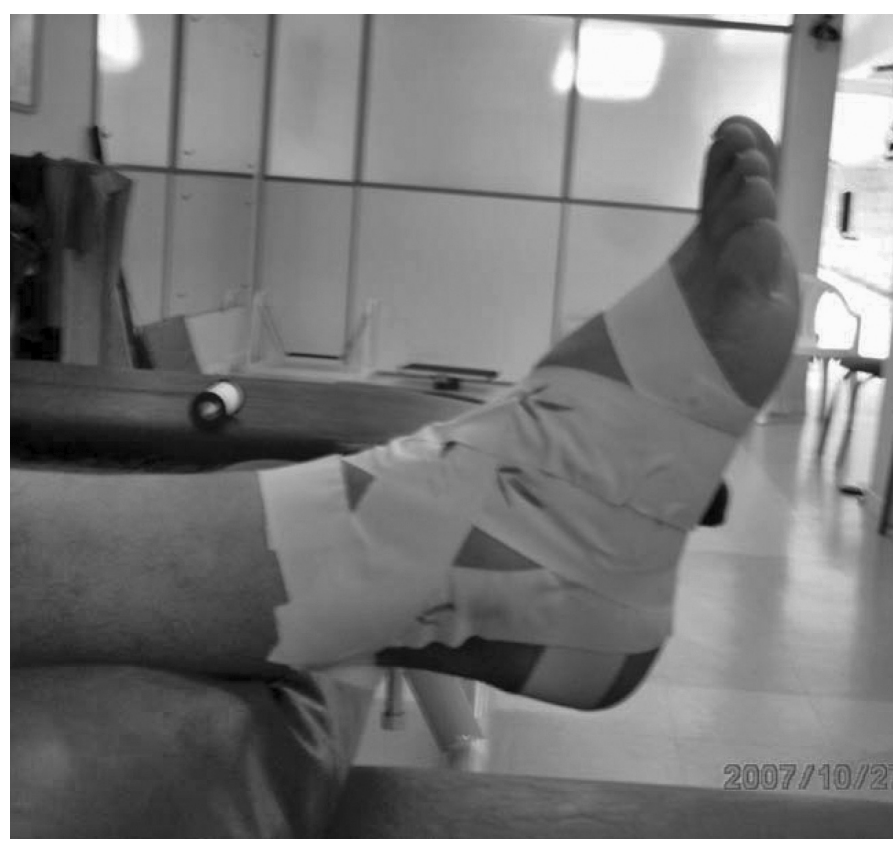

Figura 1. Bandagem funcional de tornozelo.

\section{Plataforma de inversão}

Uma plataforma eletrônica capaz de permitir a inclinação lateral do pé em $30^{\circ}$ (no plano frontal) foi utilizada na pesquisa com o objetivo de simular um movimento semelhante ao que ocorre durante uma entorse lateral de tornozelo, para a verificação dos sinais eletromiográficos provenientes da ação da musculatura fibular. Esta plataforma, apresentada na figura 2, foi construída em madeira com adaptação de um sistema eletrônico, modelo utilizado por Pacheco et al..$^{(5)}$. A plataforma era composta por duas tábuas retangulares, com um eixo transversal (anteroposterior) entre elas. Cada uma das metades possibilitava que a sua extremidade, ou borda lateral, sofresse deslocamentos em uma amplitude de $30^{\circ}$, que ia desde a posição horizontal até o contato da plataforma com a base, semelhante aos estudos de Karlsson et al. ${ }^{(10)}$, Löfvenberg et al. ${ }^{(19)}$ e Konradsen e Ravn ${ }^{(20)}$. Esse ângulo de $30^{\circ}$ foi medido e confirmado por um sistema de medição composto por um condicionador de sinais de um eletrogoniômetro. Dessa forma, essas metades permitiam a "queda" do pé apoiado, sendo esta definida como a inversão do tornozelo. Sempre que uma das tábuas estivesse produzindo a inversão do pé, a outra permanecia imóvel. Um circuito eletrônico de $220 \mathrm{~V}$ foi instalado na plataforma a fim de gerar um sinal elétrico que indicava o início do movimento de inversão do tornozelo. Esse sistema servia para sincronizar os eventos de inversão do tornozelo com a ativação elétrica do músculo. Esse sinal, proveniente do circuito eletrônico, era chamado de sincronismo e era acionado pelo pesquisador por meio de botões independentes para cada lado da plataforma. Assim, o circuito permitia que o pesquisador se mantivesse afastado do sujeito para poder acionar os botões sem que o indivíduo percebesse qual tornozelo iria inverter, o que permitia medir a resposta elétrica dos músculos fibulares, sem que o sujeito usasse qualquer mecanismo de proteção (contração muscular antes da inversão) $)^{(5,25)}$.

\section{Eletromiógrafo}

Um eletromiógrafo de oito canais (Bortec Electronics Incorporation, Canadá) foi utilizado para a aquisição dos sinais eletromiográficos (EMG). Os eletrodos eram adesivos descartáveis e de superfície do tipo pediátrico. 


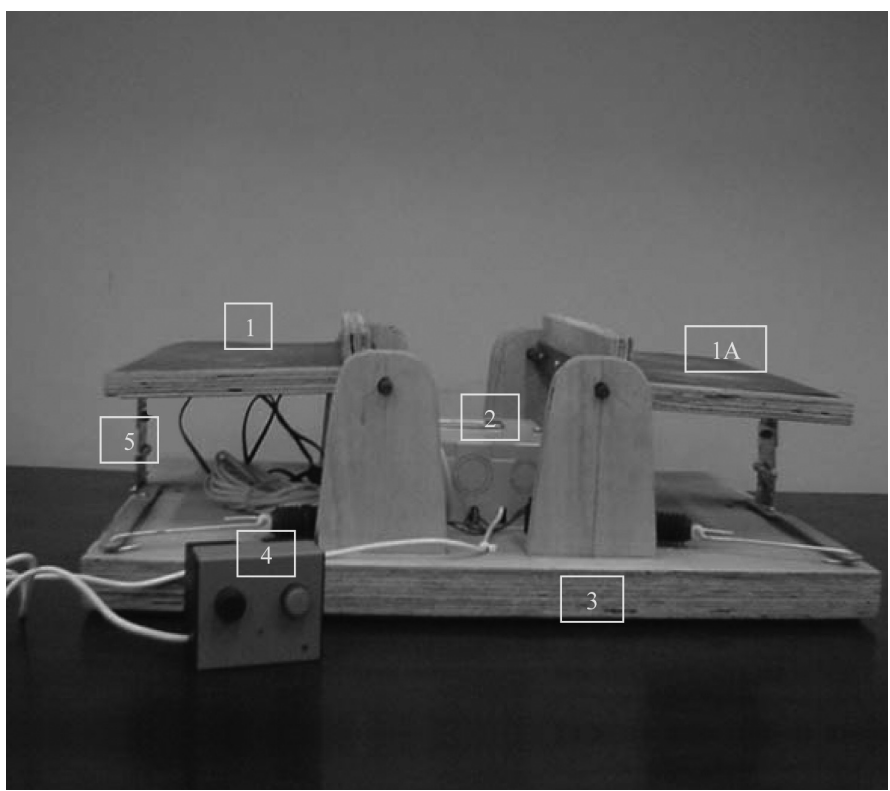

Figura 2. Plataforma eletrônica de inversão do tornozelo.

Pacheco et al. (2005).

[1] e [1A] = Tábua de queda (móvel);

[2] = Dispositivo eletrônico da plataforma (220V);

[3] = Tábua de base (fixa);

[4] = Botões de sincronismo (independente para cada metade);

[5] = Suporte metálico que, quando acionado, causa a queda da metade da plataforma.

\section{Procedimentos para coleta dos dados \\ Bandagem funcional de tornozelo}

Devido à entorse por supinação ocorrer em três eixos de movimento (adução, flexão plantar e inversão) é importante que a bandagem seja capaz de limitá-los. Em razão disto, a bandagem funcional de tornozelo do tipo bota fechada foi escolhida para a realização do teste e também por ser descrita na literatura como a técnica mais eficiente na estabilização desta articulação ${ }^{(24)}$. Por ser confeccionada de acordo com a necessidade e moldes de cada indivíduo, permite movimentos funcionais à articulação, devido ao fato de não ser rígida, mas sempre limitando graus lesivos.

\section{Plataforma de inversão e eletromiógrafo}

O sujeito era preparado para os testes realizando-se a raspagem dos pêlos (tricotomia), com lâmina descartável e pela abrasão da pele, com algodão embebido em álcool, a fim de remover as células mortas e a oleosidade no local do posicionamento dos eletrodos e com a finalidade de reduzir a impedância elétrica da pele sob os eletrodos para a coleta dos sinais eletromiográficos. A partir disso, os eletrodos eram fixados na pele e era aplicada uma leve pressão sobre eles para aumentar o contato entre o gel do eletrodo e a pele ${ }^{(5,26)}$. Os eletrodos utilizados eram adesivos descartáveis de superfície do tipo pediátrico, em configuração bipolar (um centímetro de diâmetro cada), com três centímetros de distância entre eles e foram posicionados no ventre do músculo fibular longo no sentido de seu eixo longitudinal (1/3 abaixo da cabeça da fíbula) $)^{(5,26)}$. Um eletrodo de aterramento, também adesivo e descartável, foi posicionado na tuberosidade anterior da tíbia ipsilateral, paralelo ao posicionamento dos eletrodos do fibular longo. Após esse procedimento o sujeito subia na plataforma e os sinais eletromiográficos foram coletados juntamente com o sinal da plataforma (sincronismo).
O teste foi realizado pelo sujeito em dois momentos: no primeiro, foram realizadas as inclinações laterais do tornozelo sem a aplicação da bandagem e, no segundo momento, realizaram-se as inclinações logo após a aplicação da bandagem funcional de tornozelo. Os sujeitos foram posicionados de olhos abertos sobre a plataforma e, então, foi executado um protocolo de oito inclinações aleatórias em ambos tornozelos, sendo quatro em cada, com a finalidade de evitar um possível treinamento da tarefa. Foi adotado o cuidado para que todas as inclinações fossem repentinas e inesperadas; para isto, foi colocado um aparelho tocador de MP3 player para o indivíduo escutar, com a finalidade de distrair o sujeito de modo que ele não soubesse qual seria o momento da inclinação do tornozelo e de que lado isto ocorreria ${ }^{(5)}$. Nenhum sinal de dor foi mencionado pelos sujeitos durante os testes.

\section{Análise do sinal eletromiográfico}

Os sinais eletromiográficos foram amostrados a uma frequência de $2.000 \mathrm{~Hz}$ por canal e foram filtrados com frequência mínima de $10 \mathrm{~Hz}$ e máxima de $500 \mathrm{~Hz}$. A seguir, era calculado o critério de ativação. Segundo Neptune et al.(27), quando se utilizam três desvios padrão acima da média tem-se 99,7\% de chance de considerar uma atividade diferente do repouso. Sendo assim, esse critério foi o escolhido e adotado em todas as análises do presente estudo para determinação do início da atividade elétrica até o sinal da plataforma e esta foi medida em milissegundos.

\section{Aspectos éticos e análise estatística}

Todos os procedimentos do estudo foram aprovados pelo Comitê de Ética em Pesquisa da Universidade que deu origem ao estudo, seguindo-se os preceitos éticos da Resolução 196/96 do Conselho Nacional de Saúde do Ministério da Saúde do Brasil, sob o protocolo número 1.637 e aprovados pelos participantes mediante a assinatura do Termo de Consentimento Livre Esclarecido.

O procedimento estatístico utilizado foi o teste de Wilcoxon com nível de significância de 5\%. Para a análise dos dados utilizou-se o programa SPSS for Windows (versão 13.0).

\section{RESULTADOS}

Os sujeitos tiveram média de idade de 23,3 anos, média de massa de 74,7kg, estatura média de 1,77m e IMC com média de 23,7 (kg/m²). Na tabela 1 apresenta-se a média do tempo de reação eletromiográfica do músculo fibular longo, durante a simulação da entorse lateral na plataforma, nas duas situações propostas neste estudo.

Tabela 1. Valores do tempo de reação do fibular longo nas duas situações.

\begin{tabular}{c|c|c|c}
\hline & N & Média & Desvio padrão \\
\hline Sem bandagem (ms) & 8 & 54,06 & 11,8 \\
\hline Com bandagem (ms) & 8 & 46,14 & 8,97 \\
\hline
\end{tabular}

Na figura 3 apresentam-se os valores do tempo de reação, comparados entre ambas situações. A figura demonstra haver diminuição estatisticamente significativa $(p=0,018)$ do tempo de resposta eletromiográfica do fibular longo com uso da bandagem funcional de tornozelo, sendo adotado um nível de significância de $p<0,05$, tendo um intervalo de confiança de $95 \%$. 


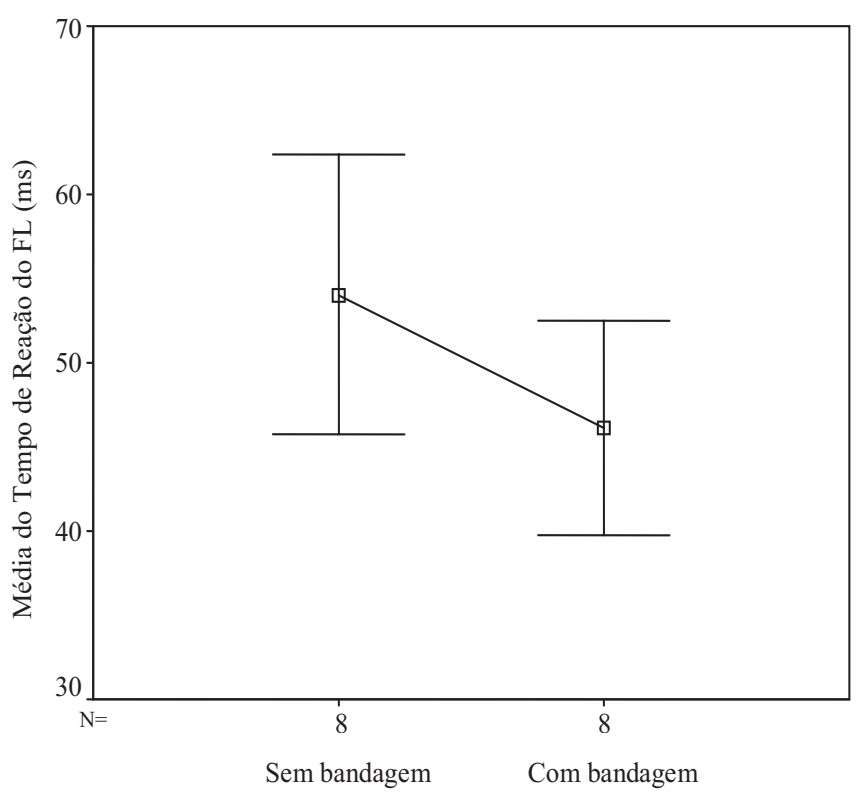

Figura 3. Diferença em ms do tempo de reação do fibular longo em ambas situações.

\section{DISCUSSÃO}

Este estudo utilizou uma plataforma eletrônica capaz de inclinar $30^{\circ}$ no plano frontal, semelhante às plataformas usadas por Karlsson et al. ${ }^{(10)}$, Löfvenberg et al. ${ }^{(19)}$ e Konradsen e Ravn ${ }^{(20)}$. Quanto à metodologia, esta foi a mesma adotada por Karlsson e Andreasson ${ }^{(13)}$ e Konradsen e Ravn ${ }^{(20)}$, que definiram o tempo de reação dos fibulares como a diferença entre o começo do movimento de inclinação da plataforma e a primeira atividade elétrica muscular. Mesmo que nossa amostra tenha sido composta por um número reduzido de indivíduos, foram encontrados valores de tempo de reação do fibular longo de acordo com o que consta na literatura. Autores, encontraram valores de normalidade para o tempo de reação fibular após inversão repentina do tornozelo na plataforma de inclinação lateral de $30^{\circ}$, que variavam de $49 \mathrm{~ms}$ a $90 \mathrm{~ms}$ em tornozelos saudáveis, indo ao encontro dos achados desta pesquisa, que teve média de $54 \mathrm{~ms}( \pm 11,8)$ de tempo de resposta do fibular longo ${ }^{(8,9)}$

Os implementos para tornozelo têm se mostrado tão importantes na entrada proprioceptiva ao sistema nervoso central, na atividade da musculatura eversora ${ }^{(12,13,16)}$ quanto já era esperado na limitação do movimento de inversão do tornozelo ${ }^{(12,24,25)}$. Em seu estudo, Heit et al. ${ }^{(18)}$ notaram que o taping e o bracing (lace up) melhoraram significativamente a habilidade dos sujeitos em reproduzir ativamente um angulo específico da articulação do tornozelo em flexão plantar. Acredita-se que isto tenha ocorrido devido à propriedade que a fita adesiva tem de realçar o senso de posição no movimento de inversão. Glick et al.(29) foram os primeiros a apresentar evidências de relacionamento entre a atividade da musculatura fibular e a bandagem de tornozelo. Usando a eletromiografia, estes encontraram que o fibular curto foi ativado por período de tempo maior no final da fase do balanço durante a marcha, com a utilização do taping. Springings et al. ${ }^{(30)}$ também fizeram uso da eletromiografia para avaliar o efeito da fita sobre a ativação da musculatura fibular, eles esperavam que esta aliviasse a tensão nas estruturas anatômicas laterais do tornozelo e, assim, terminaria por diminuir a ativação dos músculos eversores durante a inversão repentina do tornozelo. Contrário a esta expectativa, o taping não foi capaz de impedir que a musculatura eversora fosse ativada de forma vigorosa.

Karlsson e Andreasson ${ }^{(13)}$ examinaram o efeito do taping de tornozelo no tempo de reação dos fibulares medido pela eletromiografia após a inversão repentina do tornozelo. O tempo de reação mostrou-se significativamente mais lento nos tornozelos instáveis de 20 indivíduos com instabilidade unilateral, do que quando comparados aos tornozelos estáveis contralaterais. O uso do taping de tornozelo fez o tempo de reação diminuir de forma significativa, embora este valor não tenha atingido ao valor dos tornozelos estáveis. O maior ganho em relação à diminuição do tempo de reação se viu nos tornozelos com maior grau de instabilidade mecânica. Nosso estudo também encontrou melhora significativa na resposta do músculo fibular longo com a aplicação da fita adesiva, quando comparado com a situação sem a fita. Acreditamos que esta meIhora se deva ao íntimo contato da bandagem com os receptores cutâneos do tornozelo, aumentando a ativação do reflexo fibular, com consequente ganho proprioceptivo e, assim, aumenta a habilidade dos sujeitos em resposta a situações súbitas de entorses.

É importante ressaltar que a bandagem, logo que aplicada, tem efeito por tempo determinado e tudo depende do local de aplicação e qual atividade está sendo exigida com a aplicabilidade desta bandagem. Assim sendo, salientamos para futuras pesquisas que venham reproduzir a metodologia deste estudo, que não é possível afirmar que o efeito proprioceptivo será mantido por períodos longos, como na prática esportiva, pois a fita perde sua propriedade restritiva. Recomendamos a aplicabilidade correta da fita e a execução do teste imediatamente.

Purcell et al. ${ }^{(31)}$ encontraram que, após 30 minutos de exercícios, a fita perde suas propriedades de restrição. Para minimizar este efeito, os autores sugerem a reaplicação da bandagem elástica. Também Abian-Vicén et al. ${ }^{(32)}$ compararam a bandagem elástica com a não elástica após 30 minutos de exercícios, em que a bandagem elástica teve menor perda de restrição e maior conforto quando comparada a bandagem inelástica.

\section{CONCLUSÃO}

O estudo demonstrou diminuição no tempo de reação do fibular longo de indivíduos sadios com o uso da bandagem funcional de tornozelo quando submetidos à inclinação lateral súbita do tornozelo. Acreditamos que a melhora do tempo de reação muscular se deva ao íntimo contato da bandagem com os receptores cutâneos do tornozelo, aumentando a ativação do reflexo fibular, com consequente ganho proprioceptivo e, assim, aumentando a habilidade dos sujeitos em responder a situações súbitas de entorses. A literatura ainda é escassa no que se refere à metodologia proposta nesta pesquisa, porém estudos ${ }^{(33-35)}$, na sua maioria, corroboram com a ideia de que qualquer implemento para tornozelo não acarreta prejuízo na propriocepção articular.

Todos os autores declararam não haver qualquer potencial conflito de interesses referente a este artigo. 


\section{REFERÊNCIAS}

1. Bahr R, Karlsen R, Lian O. Incidence and mechanisms of acute ankle inversion injuries in volleyball. A retrospective cohort study. Am J Sports Med 1994:22:595-600.

2. Thacker SB, Stroup DF, Branche CM, Gilchrist J, Goodman RA, Weitman EA. The prevention of ankle sprains in sports. A systematic review of the literature. Am J Sports Med 1999;27:753-60.

3. Ekstrand J, Troop H. The incidence of ankle sprains in soccer. Foot Ankle 1990;11:41-4.

4. Brunt D, Andersen JC, Huntsman B, Reinhert LB, Thorell AC, Sterling JC. Postural responses to latera perturbation in healthy subjects and ankle sprain patients. Med Sci Sports Exerc 1992;24:171-6.

5. Pacheco AM, Vaz M, Pacheco I. Avaliação do tempo de resposta eletromiográfica em atletas de voleibol e não atletas que sofreram entorse de tornozelo. Rev Bras Med Esporte 2005:11:325-30.

6. Konradsen L, Voigt M, Højsgaard C. Ankle inversion injuries: the role of the dynamic defense mechanism. Am J Sports Med 1997:25:54-8.

7. Johnson $\mathrm{MB}$, Johnson $\mathrm{CL}$. Electromyographic response of peroneal muscles in surgical and nonsurgica injured ankles during sudden inversion. J Orthop Sports Phys Ther 1993;18:497-501.

8. Konradsen L, Olesen S, Hansen HM. Ankle sensoriomotor control and eversion strength after acute ankle inversion injuries. Am J Sports Med 1998;26:72-7.

9. Freeman MAR. Instability of the foot after injuries to the lateral ligament of the ankle. J Bone Joint Surg BR 1965;47:669-7.

10. Karlsson J, Peterson L, Andreasson G, Högfors C. The unstable ankle: a combined EMG and biomechanical modeling study. Int J Sports Biomech 1992;8:129-44.

11. Jerosch J, Prymka M. Proprioception and joint stability. Knee Surg Sports Traumatol Arthrosc 1996:4:171-9.

12. Alt W, Lohrer H, Gollhofer A. Functional properties of adhesive ankle taping: neuromuscular and mechanical effects before and after exercise. Foot Ankle Int 1999;20:238-45

13. Karlsson J, Andreasson GO. The effect of ankle support in chronic lateral ankle joint instability: an electromyographic study. Am J Sport Med 1992;20:257-61.

14. Wilkerson GB. Biomechanical and neuromuscular effects of ankle taping and bracing. J Athl Train 2002;37(4):436-45

15. Garrick JG, Requa RK. Role of external support in the prevention of ankle sprains. Med Sci Sports 1973;5:200-3.

16. Lohrer H, Alt W, Gollhofer A. Neuromuscular properties and functional aspects of taped ankles. Am J Sports Med 1999;27:69-75.

17. Karlsson J, Sward L, Andreasson GO. The effect of taping on ankle stability: practical implications. Sports Med 1993;16:210-5.

18. Heit EJ, Lephart SM, Rozzi SL. The effect of ankle bracing and taping on joint position senses in the stable ankle. J Sport Rehabil 1996:5:206-13.
19. Löfvenberg R, Kärrholm J, Sundelin G, Ahlgren O. Prolonged reaction time in patients with chronic lateral instability of the ankle. Am J Sports Med 1995;23:414-7.

20. Konradsen L, Ravn JB. Ankle instability caused by prolonged peroneal reaction time. Acta Orthop Scand 1990:51:388-90.

21. Gruneberg C, Nieuwenhuijzen PH, Duysens J. Reflex responses in the lower leg following landing impact on an inverting and non-inverting platform. J Physiol 2003; 550(Pt 3):985-93.

22. Prymka M, Schmidt K, Jerosch J. Proprioception in patients suffering from chondropathia patellae. Int J Sports Med 1998;19:560

23. Perlau R, Frank C, Fick G. The effect of elastic bandages on human knee proprioception on the uninjured population. Am J Sports Med 1995;23:251-5

24. Vaes $\mathrm{P}$, Boeck $\mathrm{H}$, Handelberg F, Opdecam P. Comparative radiological study of the influence of ankle joint strapping and taping on ankle stability. J Orthop Sports Phys Ther 1985;7:110-4

25. Vaes $P$, Duquet $W$, Van Gheluwe B. Peroneal reaction times and eversion motor response in healthy and unstable ankles. J Athl Train 2002;37:475-80.

26. Basmajian JV, De Luca CJ. Description and analysis of the EMG signal. Muscles alive: their function revealed by electromyography. Baltimore, Williams and Wilkins: John Butler, 1985;19-167.

27. Neptune RR, Kautz AS, Hull ML. The effect of pedaling rate on coordination in cycling. J Biomech 1997:30:1051-8.

28. Löfvenberg R, Karrholm J, Sundelin G, Lephart SM, et al. Proprioceptive reaction in the healthy and chronically unstable ankle joint. The role of proprioception in the management and rehabilitation of athletic injuries. Sportverletzung Sportschaden 1996:10:79-83.

29. Glick JM, Gordon RB, Nishimoto D. The prevention and treatment of ankle injuries. Am J Sports Med 1976;4:136-41

30. Sprigings EJ, Pelton JD, Brandell BR. An EMG analysis of the effectiveness of external ankle support during sudden ankle inversion. Can J Appl Sports Sci 1981;6:72-5.

31. Purcell SB, Schuckman BE, Docherty CL, Schrader J, Poppy W. Differences in ankle range of motion before and after exercise in 2 tape conditions. Am J Sports Med 2009;37:383-9.

32. Abián-Vicén J, Alegre L, Fernández-Rodríguez J, Aguado X. Prophylactic ankle taping: elastic versus inelastic taping. Foot \& Ankle Intern 2009;30:218-25

33. Gribble P, Radel S, Armstrong C. The effects of ankle bracing on the activation of the peroneal muscles during a lateral shuffling movement. Phys Ther Sport 2006;7:14-21.

34. Cordova ML, Ingersoll CD. Peroneus longus stretch reflex amplitude increases after ankle brace application. Br J Sports Med 2003;37:258-62.

35. Shima N, Maeda A, Hirohashi K. Delayed latency of peroneal reflex to sudden inversion with ankle taping or bracing. Int J Sports Med 2005:26:476-80. 